\title{
August 2015 Imaging Case of the Month
}

\author{
Michael B. Gotway, MD \\ Department of Radiology \\ Mayo Clinic Arizona \\ Scottsdale, AZ
}

Clinical History: A 69-year-old man with a history of hairy cell leukemia, in remission, and "smoldering" multiple myeloma (IgG kappa and M-spike with IgM kappa light chain), as well as obstructive sleep apnea treated with continuous positive airway pressure, presents with slowly progressive dyspnea, occasional cough (sometimes productive of yellow sputum) and fatigue, the latter associated with difficulty sleeping and daytime somnolence. Overnight oximetry disclosed $75 \%$ of the patient's oxygen saturations were less than $90 \%$. He is a former smoker (2.5 cigarettes / day for 15 years), but uses cannabis chronically. A chest radiograph (Figure 1) was performed.

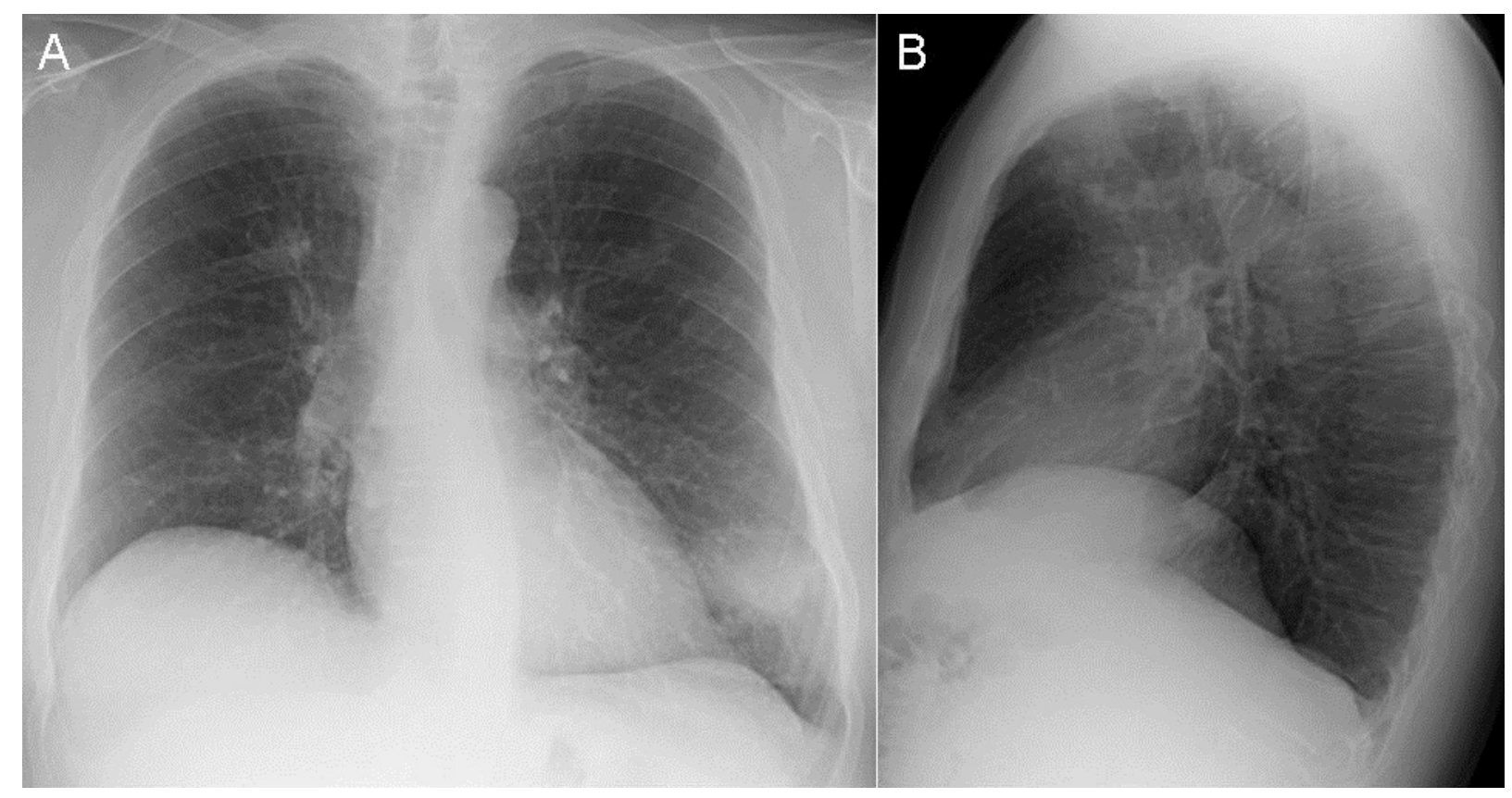

Figure 1. Frontal $(A)$ and lateral $(B)$ chest radiography.

Which of the following statements regarding the chest radiograph is most accurate?

1. The frontal chest radiograph shows abnormal mediastinal contours

2. The frontal chest radiograph shows basal predominant fibrotic abnormalities

3. The frontal chest radiograph shows bilateral nodules and masses

4. The frontal chest radiograph shows large lung volumes with a cystic appearance

5. The frontal chest radiograph shows no abnormal findings 


\section{Correct!}

3. The frontal chest radiograph shows bilateral nodules and masses

Frontal chest radiography shows a poorly defined right suprahilar nodule as well as a noncalcified mass at the left base, in the anterior portion of the left lower lobe. There is no evidence to suggest fibrotic lung disease or cystic abnormalities. No clearly abnormal mediastinal contours are evident.

What is the most appropriate next course of action?

1. ${ }^{18}$ FDG-PET scan

2. Decubitus chest radiography

3. Obtain prior imaging to determine if the opacities at chest radiography show long-term stability

4. Thoracic MRI

5. Ventilation / perfusion (V/Q) scintigraphy 


\section{Correct! \\ 3. Obtain prior imaging to determine if the opacities at chest radiography show long-term stability}

It is always advisable to make every effort to review prior imaging, particularly when abnormalities are detected at thoracic imaging studies. For example, the detection of a noncalcified nodule in an adult patient raises the possibility of lung malignancy; however, if such a lesion is shown to be stable in size for a number of years, the possibility of malignancy is effectively excluded without need for additional exposure to ionizing radiation and associated expense. Ventilation / perfusion scintigraphy is useful for the detection of pulmonary embolism as well as assessment of differential lung perfusion and for shunt detection, but it is unlikely that the opacities detected at chest radiography are due to emboli. Thoracic MRI can be useful for evaluation of some mediastinal and chest wall abnormalities, and, in selected patients, to assist in lung cancer staging, but is not preferred for assessment of lung parenchymal abnormalities. Decubitus chest radiography is useful for the detection of both pleural effusion and pneumothorax, but neither are considerations in this case. Finally, ${ }^{18}$ FDG-PET scan could prove useful for evaluation of the abnormalities detected at chest radiography in this patient, but use of ${ }^{18}$ FDG-PET is somewhat premature at this point; typically, thoracic CT will be employed for characterization of lung parenchymal abnormalities before the decision to perform ${ }^{18} \mathrm{FDG}-\mathrm{PET}$ is considered.

Clinical Course: No prior imaging was available for direct evaluation, but the report of a thoracic CT from 3 years earlier at another institution indicated the presence of emphysema, some mild basilar fibrotic change, and some mild peribronchial and mediastinal lymph node enlargement, the latter attributed to the patient's chronic lymphocytic leukemia. A repeat contrast-enhanced thoracic CT (Figure 2) was performed.

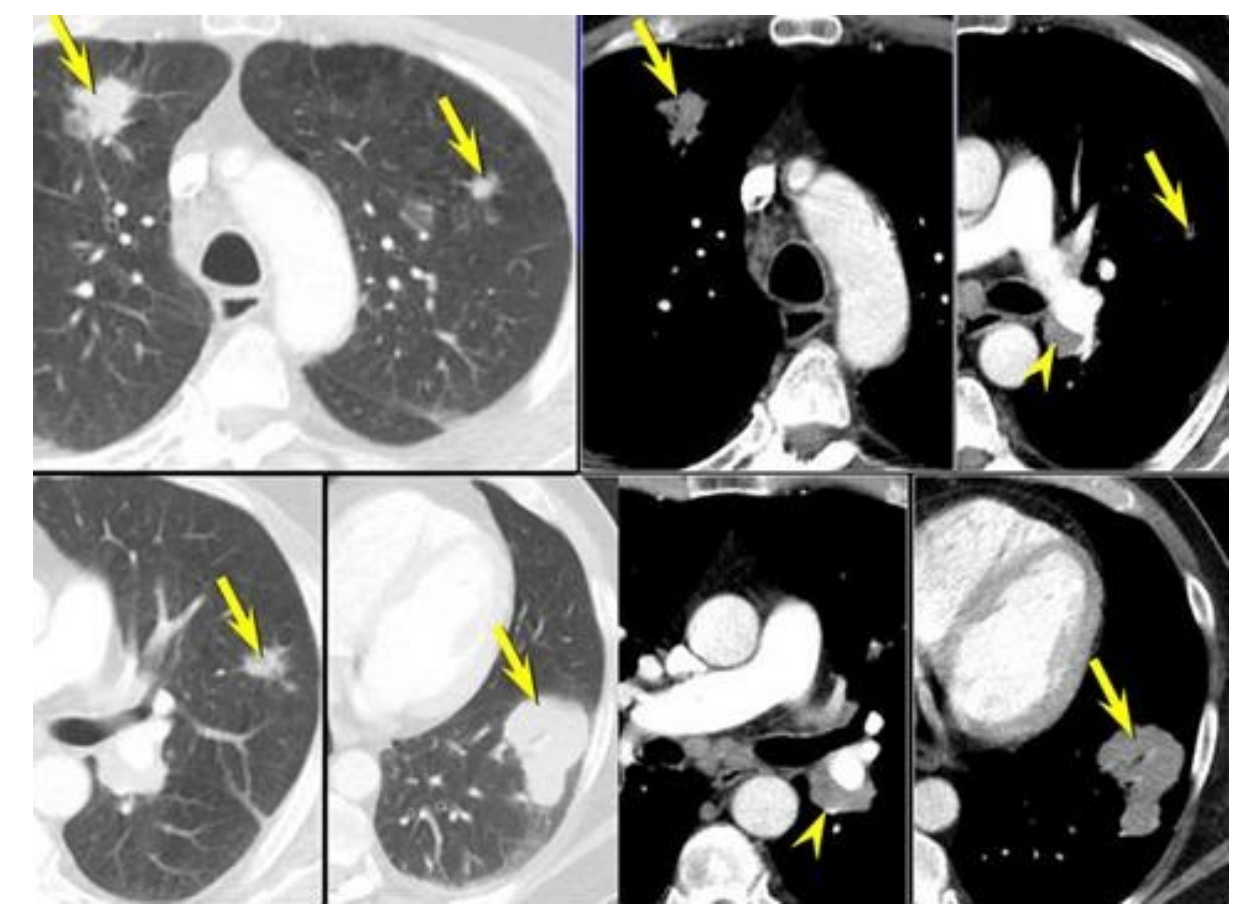

Figure 2. Contrast-enhanced axial thoracic CT displayed in lung windows (3 panels on the left ) and soft tissue windows (4 panels on the right). 
Regarding this examination, which of the following is correct?

1. Thoracic CT shows a dominant anterior mediastinal mass

2. Thoracic $\mathrm{CT}$ shows multiple small pulmonary cysts

3. Thoracic CT shows multiple, bilateral non-calcified pulmonary nodules and peribronchial and mediastinal lymph node enlargement

4. Thoracic CT shows nodular pleural thickening

5. Thoracic CT shows numerous cavitary nodules 


\section{Correct! \\ 3. Thoracic CT shows multiple, bilateral non-calcified pulmonary nodules and peribronchial and mediastinal lymph node enlargement}

The contrast-enhanced CT shows multiple, bilateral non-calcified pulmonary nodules and peribronchial and mediastinal lymph node enlargement. The peribronchial lymphadenopathy is best visualized on the left, and the largest pulmonary lesion, non-calcified, is located in the anterior basal left lower lobe, abutting the left major fissural surface. The nodules ad masses are solid; no evidence of cavitation is seen. The lucency seem within the larger lesions represent air bronchograms, not necrosis with cavitation. Emphysema is present, but there is no evidence of cystic lung disease, nor are significant pleural abnormalities present. The anterior mediastinum shows a subcentimeter lymph node, but no dominant mass. Middle mediastinal abnormalities- presenting paratracheal and left tracheobronchial angle lymphadenopathy- are present, with a prominent anterior mediastinal lymph node, but no dominant mass is seen.).

Which of the following is the most appropriate next step for the further evaluation of this patient?

1. ${ }^{18} \mathrm{FDG}-\mathrm{PET}$

2. ${ }^{68} \mathrm{Ga}$-citrate scintigraphy

3. Contrast-enhanced thoracic CT using a pulmonary nodule protocol

4. Serial CT assessment for nodule growth

5. Thoracic MRI 


\section{Correct! \\ 1. ${ }^{18}$ FDG-PET}

A contrast-enhanced thoracic CT scan using a nodule enhancement protocol can be useful for the assessment of non-calcified, indeterminate pulmonary nodules- the lack of contrast enhancement exceeding 15 Hounsfield units at any one of 4, 1-minute intervals following intravenous contrast administration, compared to the unenhanced nodule attenuation, renders the nodule indeterminate for malignancy, but lack of such enhancement is a strong predictor that the nodule is benign. This protocol, however, is typically employed for solitary indeterminate nodules ( $\leq 3 \mathrm{~cm}$ size), not multiple nodules or pulmonary masses $(>3 \mathrm{~cm}$ size), the latter largely due to the high prevalence of malignancy in lesions of this size.

Thoracic MRI is not typically employed for the evaluation of pulmonary parenchymal abnormalities, particularly if contrast-enhanced thoracic CT has already been obtained. ${ }^{68} \mathrm{Ga}-$ citrate scintigraphy has relatively few thoracic applications, but may occasionally be used for the assessment of diffuse lung abnormalities, but is not typically employed for nodules or masses. Finally, serial thoracic CT is useful for the assessment of small pulmonary nodules, to determine if intervention is required; such an approach is not useful when large nodules or frank pulmonary masses are present. ${ }^{18} \mathrm{FDG}-\mathrm{PET}$ scan can be useful when multiple indeterminate pulmonary nodules, or even masses, are found as a means of prioritizing interventions or for the detection of sites of unsuspected disease that may more be more amenable to tissue sampling than pulmonary nodules.

The patient underwent further evaluation with ${ }^{18}$ FDG-PET scanning (Figure 3).

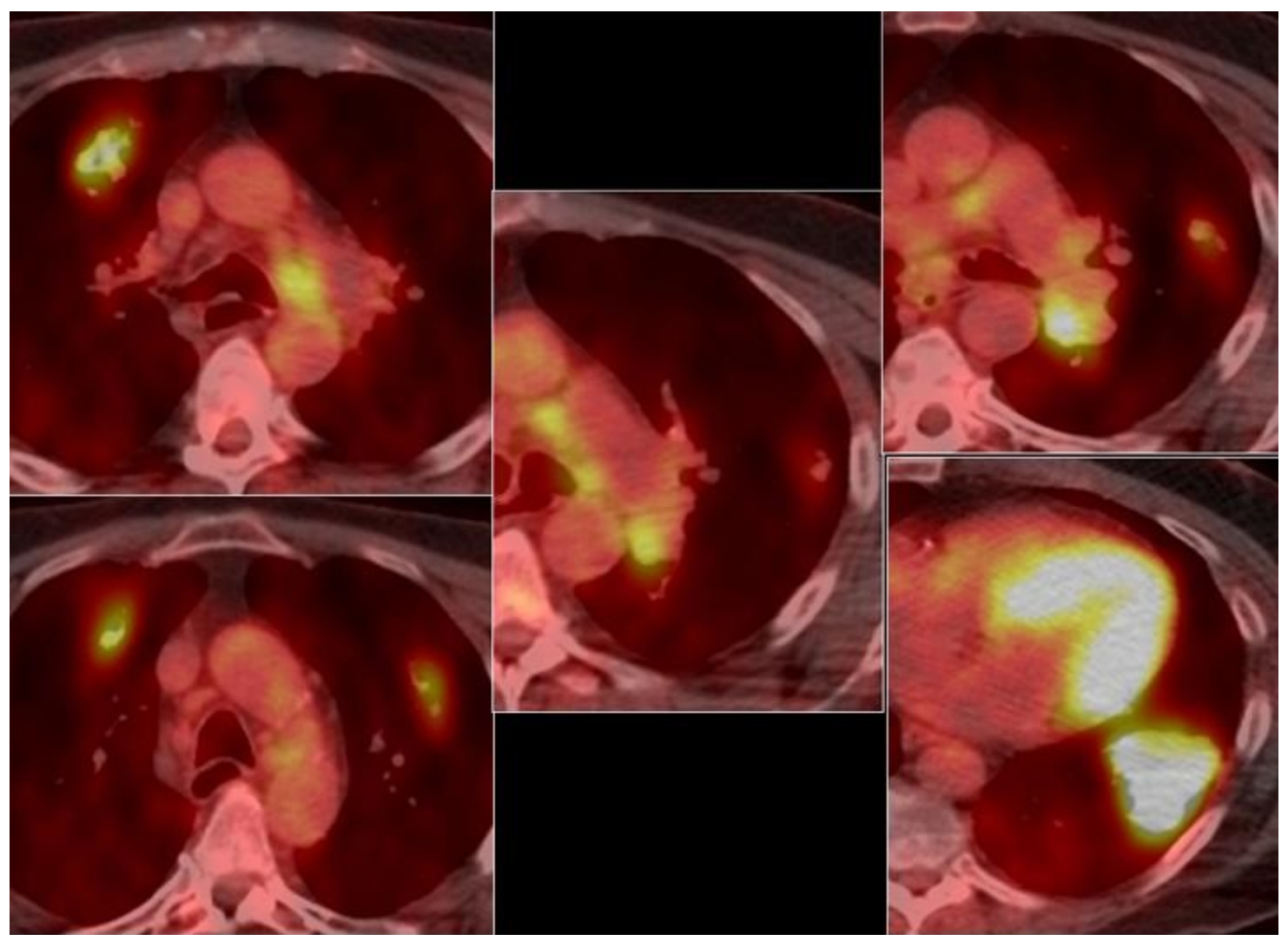

Figure 3: Axial ${ }^{18}$ FDG-PET scanning. 
Which of the following regarding this examination is correct?

1. The ${ }^{18}$ FDG-PET scan shows elevated glucose utilization within peribronchial and mediastinal lymph nodes but not within the lung parenchymal abnormalities

2. The ${ }^{18}$ FDG-PET scan shows elevated glucose utilization within peribronchial and mediastinal lymph nodes as well as the lung parenchymal abnormalities

3. The ${ }^{18}$ FDG-PET scan shows elevated glucose utilization within the peripheral portions of the lung parenchymal abnormalities, but with central photopenic ("cold") defects

4. The ${ }^{18}$ FDG-PET scan shows elevated glucose utilization within the lung parenchymal abnormalities but not within the peribronchial and mediastinal lymph nodes

5. The ${ }^{18}$ FDG-PET scan shows no abnormalities; areas of apparent tracer utilization can be explained by normally metabolically active tissues, such as brown fat 


\section{Correct! \\ 2. The 18FDG-PET scan shows elevated glucose utilization within peribronchial and mediastinal lymph nodes as well as the lung parenchymal abnormalities}

The ${ }^{18}$ FDG-PET scan shows elevated glucose utilization within peribronchial and mediastinal lymph nodes as well as the lung parenchymal abnormalities. The areas of abnormal tracer utilization do not correspond to areas of normally metabolically active tissue, such as brown fatsuch "normally" metabolically active tissues are often seen in the base of the neck near the cervical-thoracic junction and occasionally within the inter-atrial septum, as well as the left ventricular myocardium (the latter is visible in Figure 3). The areas of abnormal tracer utilization show fairly homogeneous, intense activity; no central photopenic defects [which may be seen when a metabolically lesion is centrally necrotic] are present.

Which of the following represents an appropriate step for the evaluation of this patient?

1. Bronchoscopy with transbronchial biopsy

2. Mini-thoracotomy

3. Percutaneous fine needle aspiration biopsy

4. Video-assisted thoracoscopic surgical biopsy

5. 1 or 3 


\section{Correct!}

\section{1 or 3}

Either percutaneous fine needle aspiration biopsy or bronchoscopy with transbronchial biopsy could be performed for this patient, and both would offer a high likelihood for obtaining a diagnosis. Bronchoscopic procedures have the advantage of fewer complications than percutaneous needle procedures, although the latter has the advantage of obtaining a relatively larger tissue sample when core biopsy is performed. Note that core biopsy was not mentioned

as part of choice $d$; only fine needle aspiration biopsy was indicated in the question. Core biopsy can be performed as part of percutaneous transthoracic biopsy procedures, but the operator must choose to perform the core procedure in addition to, or in place of, the fine needle aspiration. Fine needle aspiration procedures provides material for cytology, which is often sufficient for the diagnosis of carcinoma and sometimes sarcoma, as the abnormal cells may be visible with this procedure. However, the architecture of the tissue sample is not maintained with fine needle aspiration samples, as it is with core biopsy, and therefore a histopathological diagnosis may not be possible for lesions sampled using fine needle aspiration. Some conditions require histopathology for a confident diagnosis, including some malignancies and, notably, fibrous lesions and some benign causes of lung nodules. As such, many operators choose to perform both fine needle aspiration and core biopsy with every percutaneous procedure, particularly if on-site cytological evaluation is not available at the time of the procedure. Video-assisted thoracoscopic (VATS) biopsy is not a "wrong" choice- the left lower lobe lesion could be sampled with this technique, given its proximity to the pleural surface, and the procedure provides ample tissue for diagnosis. If there are only one lesion in the lung, VATS could be the best choice- if cancer we found, an operation could then be performed for staging at a single sitting, rather than one or more minimally invasive procedures followed by a surgical procedure. However, in this case, given the multiplicity of findings, a minimally invasive approach to obtaining the diagnosis may be advisable. Finally, mini-thoracotomy is needlessly invasive at this point, and is typically reserved for situations in which less invasive attempts at diagnosis have not been successful.

Percutaneous fine needle aspiration biopsy was performed, and showed a blood-diluted specimen with benign bronchoepithelial cells as well as reactive bronchoalveolar cells with scattered multinucleated giant cells and chronic inflammation; no specific diagnosis was offered.

Which of the following represents an appropriate step for the evaluation of this patient?

1. Bronchoscopy with transbronchial biopsy

2. Mini-thoracotomy

3. Percutaneous fine needle aspiration and core biopsy

4. Video-assisted thoracoscopic surgical biopsy

5. All of the above except 2 


\section{Correct! \\ 5. All of the above except 2}

Again, either percutaneous fine needle aspiration biopsy or bronchoscopy with transbronchial biopsy could be performed for this patient, and both would offer a reasonable likelihood for obtaining a diagnosis. In the context of a repeat procedure, however, if percutaneous sampling is pursued, core biopsy should be performed, with or without, fine needle aspiration biopsy. A video-assisted thoracoscopic procedure is now appropriate, despite its more invasive nature than either percutaneous sampling or bronchoscopy, given that a previous percutaneous tissue sampling procedure has been performed already and did not successfully obtain a diagnosis for this patient. A mini-thoracotomy still remains needlessly invasive at this point. Ultimately, the choice of procedure in this circumstance must be carefully weighed and depends on a balance between procedural risk tolerance, available expertise, and patient / physician preference.

The patient underwent repeat transthoracic percutaneous fine needle aspiration and core biopsy (Figure 4), which showed an infiltrate of plasma cells within a fibrous background. Immunostains showed positivity for DC138 and kappa light chain restriction, and the plasma cells stained positively for cyclin D1.

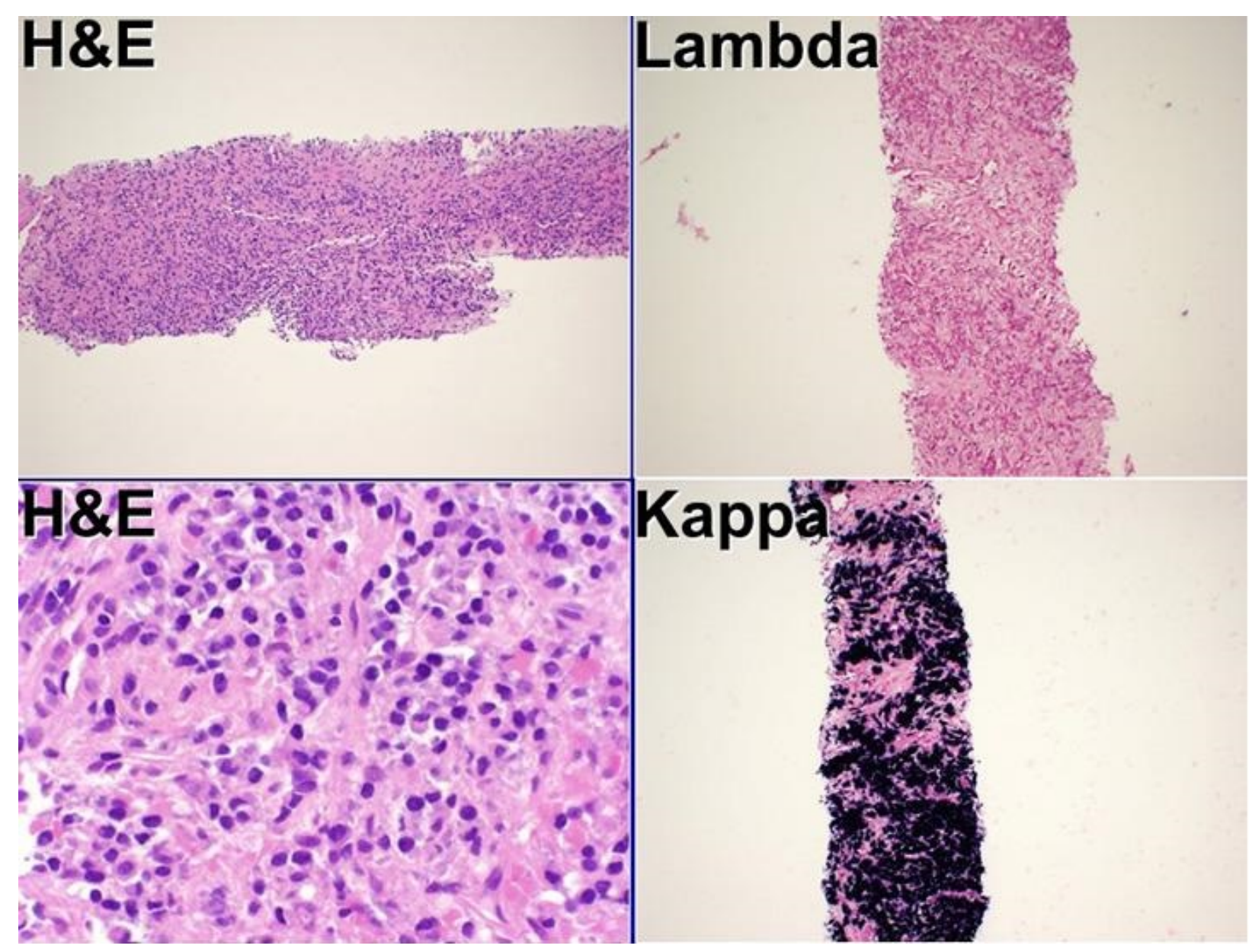

Figure 4. Histopathological specimen obtained from core biopsy shows plasma cells embedded within a background fibrous stroma. Immunostaining shows positive staining for kappa, but not lambda, light chains. 
Which of the following is the most likely consideration for the patient's condition given the data thus far?

1. Amyloidosis

2. Hairy cell leukemia in the lung

3. Lymphocytic interstitial pneumonia

4. Plasmacytoma

5. Pulmonary vasculitis 


\section{Correct! \\ 4. Plasmacytoma}

The histopathological findings are consistent with the diagnosis of plasmacytoma. No features of amyloidosis were noted, and no capillaritis was seen to suggest pulmonary vasculitis. Flow cytometry did not disclose the presence of lymphoma / leukemia. The histopathological pattern was not consistent with the cellular interstitial inflammatory infiltration typically seen with lymphocytic interstitial pneumonia.

Diagnosis: Plasmacytoma in the lung

\section{References}

1. Ooi GC, Chim JC, Au WY, Khong PL. Radiologic manifestations of primary solitary extramedullary and multiple solitary plasmacytomas. AJR Am J Roentgenol. 2006;186 (3):821-7. [CrossRef] [PubMed]

2. Ujiie H, Okada D, Nakajima Y, Yoshino N, Akiyama H. A case of primary solitary pulmonary plasmacytoma. Ann Thorac Cardiovasc Surg. 2012;18(3):239-42. [CrossRef] [PubMed]

3. Shah BK, Shrestha A. Pulmonary plasmacytoma. J Thorac Oncol. 2012; 7(5):923. [CrossRef] [PubMed]

4. Niitsu N, Kohri M, Hayama M, Nakamine H, Nakamura N, Bessho M, Higashihara M. Primary pulmonary plasmacytoma involving bilateral lungs and marked hypergammaglobulinemia: differentiation from extranodal marginal zone B-cell lymphoma of mucosa-associated lymphoid tissue. Leuk Res. 2005; 29(11):1361-4. [CrossRef] [PubMed]

5. Kaneko Y, Satoh H, Haraguchi N, Imagawa S, Sekizawa K. Radiologic findings in primary pulmonary plasmacytoma. J Thorac Imaging. 2005; 20(1):53-4. [CrossRef] [PubMed] 